peptides from a mutant cell line: a second pathway of antigen presentation. Science. 255:1264-1266.

9. Wei, M.L., and Cresswell, P. 1992. HLA-A2 molecules in an antigen-processing mutant cell contain signal sequence-derived peptides. Nature. 356:443-446.

10. Kent, S.C., et al. 2005. Expanded T cells from pancreatic lymph nodes of type 1 diabetic subjects rec ognize an insulin epitope. Nature. 435:224-228.

11. Nakayama, M., et al. 2005. Prime role for an insulin epitope in the development of type 1 diabetes in NOD mice. Nature. 435:220-223.

12. Gueguen, M., Biddison, W.E., and Long, E.O. 1994. T cell recognition of an HLA-A2-restricted epitope derived from a cleaved signal sequence. J. Exp. Med.
180:1989-1994.

13. Wolfel, C., et al. 2000. Transporter (TAP)- and proteasome-independent presentation of a melanoma-associated tyrosinase epitope. Int. J. Cancer. 88:432-438.

14. McDevitt, H.O. 1998. The role of MHC class II molecules in susceptibility and resistance to autoimmunity. Curr. Opin. Immunol. 10:677-681.

15. Goudy, K.S., and Tisch, R. 2005. Immunotherapy for the prevention and treatment of type 1 diabetes. Int. Rev. Immunol. 24:307-326.

16. Calderon, B., Suri, A., Miller, M.J., and Unanue, E.R. 2008. Dendritic cells in islets of Langerhans constitutively present beta cell-derived peptides bound to their class II MHC molecules. Proc. Natl. Acad. Sci. U. S. A. 105:6121-6126.

17. Kendall, P.L., Yu, G., Woodward, E.J., and Thomas, J.W. 2007. Tertiary lymphoid structures in the pancreas promote selection of B lymphocytes in autoimmune diabetes. J. Immunol. 178:5643-5651.

18. Ludewig, B., Odermatt, B., Landmann, S., Hengartner, H., and Zinkernagel, R.M. 1998. Dendritic cells induce autoimmune diabetes and maintain disease via de novo formation of local lymphoid tissue. J. Exp. Med. 188:1493-1501.

19. Tang, Q., et al. 2004. In vitro-expanded antigenspecific regulatory $\mathrm{T}$ cells suppress autoimmune diabetes. J. Exp. Med. 199:1455-1465

\title{
Coaxing the liver into preventing autoimmune disease in the brain
}

\author{
Brad E. Hoffman and Roland W. Herzog
}

Department of Pediatrics, University of Florida, Gainesville, Florida, USA.

\begin{abstract}
The liver has several unique immunological properties that affect $T$ cell activation and immune regulation. Recent studies have uncovered opportunities for the treatment of genetic disease by directing expression of the functional therapeutic protein to hepatocytes. In a new study in this issue of the JCI, Lüth and colleagues demonstrate that hepatic expression of a brain protein is protective against neuroinflammatory disease in a mouse model of human MS (see the related article beginning on page 3403). Suppression of autoimmunity was dependent on transgene expression in the liver and was mediated by induction of antigen-specific $\mathrm{CD}^{+} \mathrm{CD}^{+} 5^{+} \mathrm{Foxp} 3^{+}$Tregs. These findings suggest that the introduction of antigens to the liver may have potential as a preventative or therapeutic intervention for autoimmune disease.
\end{abstract}

Immune responses are substantially influenced by the context of the tissue in which they occur. For example, brain and liver have both been characterized as immuneprivileged sites. The liver is constantly exposed to food antigens and therefore must control inflammatory responses to exogenous antigens. Furthermore, injection of antigen into the portal vein can induce tolerance, and spontaneous acceptance of liver allograft accompanied by donor-specific $\mathrm{T}$ cell tolerance has been documented previously (1). The immune system in the brain parenchyma lacks dendritic cells and therefore the ability to prime $\mathrm{T}$ cells. However, $\mathrm{T}$ cell activation upon immunization at an ectopic site can cause lymphocyte infiltration and inflam-

Nonstandard abbreviations used: MBP, myelin basic protein.

Conflict of interest: The authors have declared that no conflict of interest exists.

Citation for this article: J. Clin. Invest. 118:3271-3273 (2008). doi:10.1172/JCI37079. matory responses targeting antigens in the brain (2). For example, immunization against myelin basic protein (MBP) - a protein important in the process of nerve myelination - causes EAE in certain strains of mice, and these animals have been used as a model for human MS.

On the other hand, it is also known that antigen administration to specific organs can induce suppressive immune responses that ultimately promote tolerance. Oral and nasal tolerance are extensively studied scenarios in which antigen introduced to mucosal surfaces at optimal doses causes activation of Tregs such as $\operatorname{Tr} 1$ or Th 3 cells (3-5). These subsets of CD4 ${ }^{+} \mathrm{T}$ cells can suppress immune responses by secretion of the cytokines IL-10 and TGF- $\beta$. Similarly, antigen administered to the anterior chamber of the eye, another immune-privileged site, is transported to the spleen via a macrophage-like F4/80+ cell (6). Antigen presentation by this cell type in the lymphoid tissue causes activation of $\mathrm{CD}^{+}$and $\mathrm{CD}^{+}$ Tregs and NKT cells, resulting in immune suppression. These examples illustrate how the immune system has evolved to generate cellular mediators of tolerance, which are exposed to antigens in the context of one organ and are capable of subsequently transferring tolerance to a different organ.

\section{Gene transfer to the liver induces immune tolerance}

The question of how to establish immune tolerance is critical for gene- and cell-based therapies for genetic and autoimmune diseases. Interestingly, a number of reports in recent years have shown that hepatic gene transfer can induce tolerance to a variety of therapeutic proteins (7-13). While there has been evidence of deletion and anergy of transgene product-specific $T$ cells following hepatocyte-derived gene expression, a finding key to our understanding of the mechanism of tolerance induction was the observation of $\mathrm{CD}^{+}$Treg activation (14-16). Using a $\mathrm{T}$ cell receptor transgenic model, the induction of antigen-specific $\mathrm{CD}^{+} \mathrm{CD} 25^{+} \mathrm{Foxp}^{+} \mathrm{T}$ cells by liver-directed gene transfer with an adeno-associated viral vector has been previously demonstrated (14). These induced T cells appeared phenotypically comparable to naturally occurring Tregs, which are required to prevent autoimmune disease. In other experiments, Tregs induced by hepatic adeno-associated viral gene transfer were capable of suppressing antibody formation and $\mathrm{CD}^{+}$ $T$ cell responses against a coagulation Factor IX gene product and were found to be required for tolerance induction $(14,17)$. 


\section{A EAE mouse model}

B Prevention of EAE by hepatic expression of MBP
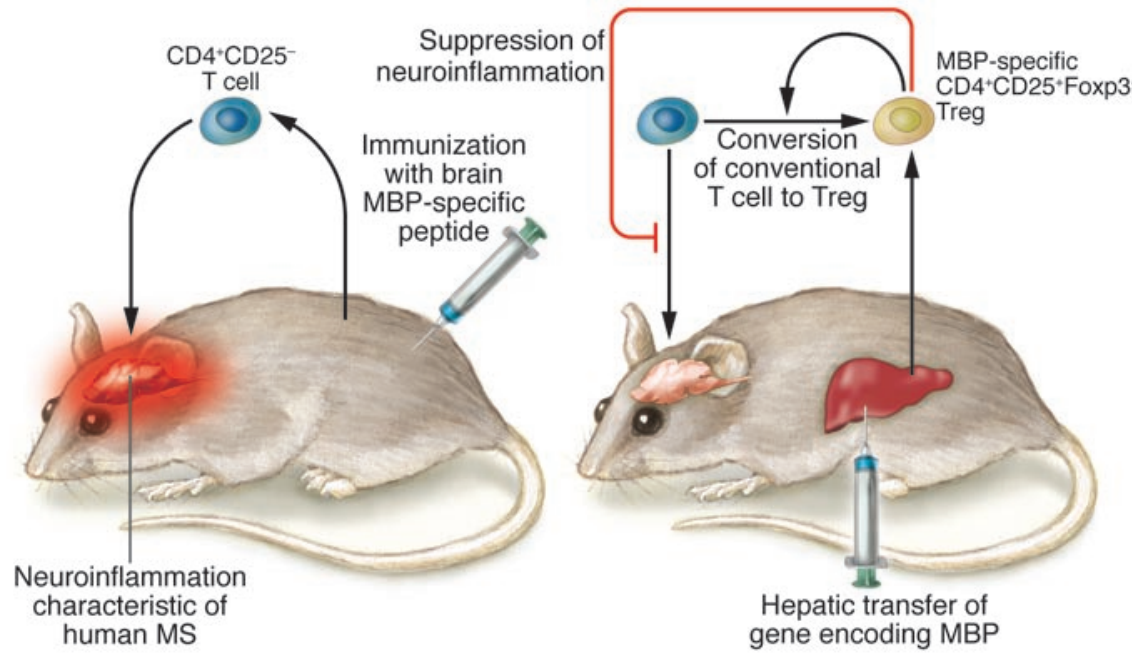

Figure 1

Prevention of EAE in mice by hepatic expression of MBP. (A) Immunization with MBP-specific peptide normally causes an inflammatory $T$ cell response in the brain in the EAE mouse model of human MS. (B) In the study by Lüth et al. in this issue of the $\mathrm{JCl}(20)$, the authors show that hepatic expression of MBP (normally expressed in the central nervous system), accomplished constitutively in liver-specific MBP transgenic mice (not shown) or transiently after gene transfer to liver cells in vivo, results in induction of MBP-specific CD4+CD25+Foxp3+ Tregs. These MBP-specific CD4 ${ }^{+} \mathrm{CD} 25^{+}$Foxp3 ${ }^{+}$Tregs suppressed effector $\mathrm{CD} 4^{+} \mathrm{CD} 25^{-} \mathrm{T}$ cells and converted conventional $\mathrm{CD} 4{ }^{+} \mathrm{CD} 25^{-} \mathrm{T}$ cells into $\mathrm{CD} 4{ }^{+} \mathrm{CD} 25^{+} \mathrm{Foxp} 3^{+}$Tregs, thereby preventing neuroinflammatory disease. Introduction of antigens to the liver may have potential as a preventative or therapeutic intervention for autoimmune disease.

Moreover, tolerance established by antigen expression in the liver was upheld when the antigen was subsequently expressed in other organs, such as skeletal muscle, or was delivered i.v. $(18,19)$. Therefore, hepatic gene expression can suppress antibody and cellular immune responses to a specific protein in extrahepatic sites. These findings have important practical implications for the treatment of genetic disease. For example, tolerized animals can now receive supplementary therapies such as enzyme replacement therapy.

\section{Tregs induced by hepatic antigen expression prevent neuroinflammatory disease}

In this issue of the JCI, Lüth et al. report exciting data that illustrate the far-reaching implications of this hepatic tolerance model (20). The authors demonstrate that expression of MBP in the livers of mice via transgenic and gene transfer approaches prevents the neuroinflammation characteristic of EAE, providing what is believed to be the first evidence that the tolerogenic potential of the liver can abrogate development of autoimmune disease even if the target antigen for the inflammatory $\mathrm{T}$ cell response is located in a distant organ, in this instance the brain (Figure 1).

Mechanistically, the authors showed again that peripheral $\mathrm{CD} 4^{+} \mathrm{CD} 25^{+} \mathrm{Foxp}^{+}$ Tregs mediated tolerance, while the results of experiments in $\mathrm{T}$ cell receptor transgenic animals showed no evidence for clonal deletion of MBP-reactive effector $\mathrm{T}$ cells, despite some level of expression in the thymus of liver MBP-transgenic mice (20). Importantly, expression of MBP in the skin did not protect against EAE, which indicates that expression of this protein in the hepatic environment is critical. In an elegant set of experiments based on adoptive transfer of Tregs and effector $\mathrm{T}$ cells labeled with fluorescent dye, the authors showed that Tregs induced by hepatic expression can turn effector $\mathrm{T}$ cells into Tregs by inducing expression of the transcription factor Foxp3. Effector $\mathrm{T}$ cells lacking TGF- $\beta$ receptor II were resistant to this infectious tolerance mechanism, indicating dependence on TGF- $\beta$ signaling. These findings are reminiscent of TGF- $\beta$-dependent suppression of $\mathrm{CD}^{+}$ $\mathrm{T}$ cells by $\mathrm{CD}^{+} \mathrm{CD} 25^{+} \mathrm{Foxp}^{3^{+}}$Tregs previously reported by Mempel, von Andrian, and colleagues (21) and further substan- tiate the importance of this cytokine for in vivo suppression.

The current study by Lüth et al. provides insight into how tolerance is manifested following initial induction of Tregs. Consistent with previously published data (12), hepatic-induced tolerance was found to be robust and not broken by immunization with antigen in complete Freund's adjuvant. In the EAE model, tolerant mice lacked the striking inflammation as well as $T$ cell and macrophage infiltrate seen in the brains of control animals after immunization with MBP (20). The present study opens the door to the treatment of autoimmune disease by antigen administration to the liver. More work will be required to test whether ongoing disease can be reversed, as opposed to prevented.

The study also raises several interesting questions about the mechanism of Treg generation, including whether antigen presentation in the liver or in a lymphoid tissue induces $\mathrm{CD}^{+}{ }^{+} \mathrm{CD} 25^{+} \mathrm{Foxp}^{+}$Tregs and which antigen-presenting cells mediate Treg induction. It should be noted that the liver represents a unique microenvironment with several specialized antigen-presenting cells, such as Kupffer cells (resident macrophages), liver sinusoidal endothelial cells, and hepatic dendritic cells, which have all been implicated in $\mathrm{T}$ cell activation and tolerance induction $(1,22,23)$. Furthermore, suppressive cytokines such as IL-10 and TGF- $\beta$ are expressed in the liver and have important roles in regulating antigen uptake and presentation as well as $\mathrm{T}$ cell activation. In the present study, suppression of MBP-specific effector $\mathrm{T}$ cells by Tregs likely occurred at the level of the draining lymph nodes of the site of immunization and perhaps in the spleen. It would be exciting if this model could be further developed to study potential interactions between ectopically induced Tregs and the immune system of the brain.

Address correspondence to: Roland W. Herzog, Cancer and Genetics Research Center, University of Florida, 1376 Mowry Road, Room 203, Gainesville, Florida 32610, USA. Phone: (352) 273-8113; Fax: (352) 273-8342; E-mail: rherzog@ufl.edu.

\footnotetext{
1. Crispe, I.N., et al. 2006. Cellular and molecular mechanisms of liver tolerance. Immunol. Rev. 213:101-118.

2. Lowenstein, P.R., Mandel, R.J., Xiong, W.D., Kroeger, K., and Castro, M.G. 2007. Immune responses to adenovirus and adeno-associated vectors used for gene therapy of brain diseases: the role of immunological synapses in understanding the cell biology of neuroimmune interactions. Curr. Gene Ther.
} 
7:347-360.

3. Fukaura, H., et al. 1996. Induction of circulating myelin basic protein and proteolipid protein-specific transforming growth factor-beta1-secreting Th3 $\mathrm{T}$ cells by oral administration of myelin in multiple sclerosis patients. J. Clin. Invest. 98:70-77.

4. Karachunski, P.I., Ostlie, N.S., Okita, D.K., and Conti-Fine, B.M. 1997. Prevention of experimental myasthenia gravis by nasal administration of synthetic acetylcholine receptor $\mathrm{T}$ epitope sequences. J. Clin. Invest. 100:3027-3035.

5. Santos, L.M., al-Sabbagh, A., Londono, A., and Weiner, H.L. 1994. Oral tolerance to myelin basic protein induces regulatory TGF-beta-secreting T cells in Peyer's patches of SJL mice. Cell. Immunol. 157:439-447

6. Streilein, J.W. 2003. Ocular immune privilege: therapeutic opportunities from an experiment of nature. Nat. Rev. Immunol. 3:879-889.

7. Brown, B.D., et al. 2007. A microRNA-regulated lentiviral vector mediates stable correction of hemophilia B mice. Blood. 110:4144-4152.

8. Cerullo, V., et al. 2007. Antigen-specific tolerance of human alpha1-antitrypsin induced by helper-dependent adenovirus. Hum. Gene Ther. 18:1215-1224.

9. Dobrzynski, E., and Herzog, R.W. 2005. Tolerance induction by viral in vivo gene transfer. Clin. Med. Res. 3:234-240.

10. Franco, L.M., et al. 2005. Evasion of immune responses to introduced human acid alpha-glucosidase by liver-restricted expression in glycogen storage disease type II. Mol. Ther. 12:876-884.

11. Herzog, R.W. 2005. Recent advances in hepatic gene transfer: more efficacy and less immunogenicity. Curr. Opin. Drug Discov. Devel. 8:199-206.

12. Mingozzi, F., et al. 2003. Induction of immune tolerance to coagulation factor IX antigen by in vivo hepatic gene transfer. J. Clin. Invest. 111:1347-1356.

13. Ziegler, R.J., et al. 2004. AAV2 vector harboring a liver-restricted promoter facilitates sustained expression of therapeutic levels of alpha-galactosidase $\mathrm{A}$ and the induction of immune tolerance in fabry mice. Mol. Ther. 9:231-240.

14. Cao, O., et al. 2007. Induction and role of regulatory CD4+CD25+ T cells in tolerance to the transgene product following hepatic in vivo gene transfer. Blood. 110:1132-1140

15. Cao, O., Furlan-Freguia, C., Arruda, V.R., and Herzog, R.W. 2007. Emerging role of regulatory T cells in gene transfer. Curr. Gene Ther. 7:381-390.

16. Dobrzynski, E., et al. 2004. Induction of antigenspecific CD4+ T-cell anergy and deletion by in vivo viral gene transfer. Blood. 104:969-977.

17. Dobrzynski, E., et al. 2006. Prevention of cytotoxic $\mathrm{T}$ lymphocyte responses to factor IX-expressing hepatocytes by gene transfer-induced regulatory T cells. Proc. Natl. Acad. Sci. U. S. A. 103:4592-4597.

18. Hoffman, B.E., et al. 2007. Muscle as a target for supplementary factor IX gene transfer. Hum. Gene Ther. 18:603-613.

19. Sun, B., et al. 2007. Enhanced response to enzyme replacement therapy in Pompe disease after the induction of immune tolerance. Am. J. Hum. Genet. 81:1042-1049.

20. Lüth, S., et al. 2008. Ectopic expression of neural autoantigen in mouse liver suppresses experimental autoimmune neuroinflammation by inducing antigen-specific Tregs. J. Clin. Invest. 118:3403-3410.

21. Mempel, T.R., et al. 2006. Regulatory T cells reversibly suppress cytotoxic $\mathrm{T}$ cell function independent of effector differentiation. Immunity. 25:129-141.

22. Knolle, P.A., and Gerken, G. 2000. Local control of the immune response in the liver. Immunol. Rev. 174:21-34.

23. Limmer, A., et al. 2000. Efficient presentation of exogenous antigen by liver endothelial cells to CD8 + $\mathrm{T}$ cells results in antigen-specific T-cell tolerance. Nat. Med. 6:1348-1354.

\section{Toward a broadly protective influenza vaccine}

\section{Peter C. Doherty ${ }^{1,2}$ and Anne Kelso ${ }^{3}$}

1Department of Microbiology and Immunology, University of Melbourne, Melbourne, Victoria, Australia. 2Department of Immunology, St. Jude Children's Research Hospital, Memphis, Tennessee, USA. ${ }^{3}$ WHO Collaborating Centre for Reference and Research on Influenza, Parkville, Victoria, Australia.

\begin{abstract}
The current inactivated influenza virus vaccines induce antibodies that protect against closely related virus strains. They do not, however, protect against antibody-escape variants of seasonal influenza A viruses or new pandemic influenza A viruses emerging from non-human reservoirs. Might boosting influenza A virus-specific $\mathrm{CD8}^{+} \mathrm{T}$ cell memory diminish the danger posed by these variant viruses? Pre-existing $\mathrm{CD8}^{+} \mathrm{T}$ cell-mediated immunity directed at peptides from conserved internal proteins of the influenza $A$ virus does not prevent infection, but it can promote early virus clearance and decrease morbidity in mice. In this issue of the JCI, Lee et al. show that people who have not been exposed to avian influenza A (H5N1) viruses have cross-reactive $\mathrm{CD8}^{+} \mathrm{T}$ cell memory to a wide range of $\mathrm{H} 5 \mathrm{~N} 1$ peptides (see the related article beginning on page 3478 ). These peptides could be used to add a CD8 ${ }^{+}$ $T$ cell component to current antibody-focused vaccine strategies with a view to reducing the impact of infection with novel influenza A viruses.
\end{abstract}

The recent spread of the extremely virulent avian influenza A subtype H5N1 viruses, herein referred to as $\mathrm{H} 5 \mathrm{~N} 1$, through Asia and to North Africa and Europe has raised serious concerns about the possibility of a novel human influenza pandemic $(1,2)$. Though the severe disease that can develop

Nonstandard abbreviations used: M1, matrix protein 1; NA, neuraminidase; NP, nucleoprotein.

Conflict of interest: The authors have declared that no conflict of interest exists.

Citation for this article: J. Clin. Invest. 118:3273-3275 (2008). doi:10.1172/JCI37232. in humans exposed to H5N1-infected birds is rare and sustained human-to-human transmission of the virus has not yet been observed, the three influenza pandemics of the 20th century were all caused by influenza A viruses that originated from birds (3). Variant influenza A (H1N1), A (H3N2), and $\mathrm{B}$ viruses also cause regular seasonal epidemics that are associated with substantial morbidity and economic loss. It is bad enough that some 250,000-500,000 (particularly elderly) people die annually from influenza, but what if we should face an event like the 1918-1919 influenza pandemic? That pan- demic killed in excess of 40 million people worldwide - before the era of rapid air travel and at a time when the global population was less than a third of that today.

\section{Limitations of current influenza vaccines}

Inactivated influenza vaccines elicit neutralizing antibody responses that provide reasonable protection against the homologous H1N1, H3N2, and B viruses (4). However, antibody-mediated selection drives changes (known as antigenic drift) in the viral HA $(\mathrm{H})$ and neuraminidase (NA; N) surface glycoproteins, which in turn dictate the frequent production of a new vaccine, sometimes as often as annually, as has been the case in each of the last five years. The WHO recommends candidate vaccine virus strains that have been identified among the collections of emerging field isolates supplied by a global network of $124 \mathrm{WHO}$ National Influenza Centers and other diagnostic laboratories and characterized by the four WHO Collaborating Centers for Influenza (in London, Atlanta, Melbourne, and Tokyo). The WHO's recommendations also inform the composition of the live vaccines produced from "cold-adapted" viruses that 